\title{
Elastic-Perfectly Plastic Contact of Rough Surfaces: An Incremental Equivalent Circular Model
}

\author{
X. M. Liang, Y. Ding, D. Yan, W. K. Yuan and G. F. Wang* \\ Department of Engineering Mechanics, SVL, Xi'an Jiaotong University, Xi'an \\ 710049, China \\ *E-mail: wanggf@mail.xjtu.edu.cn
}

\begin{abstract}
In this paper, an incremental eqivalent contact model is developed for elastic-perfectly plastic solids with rough surfaces. The contact of rough surface is modeled by the accumulation of circular contacts with varying radius, which is estimated from the geometrical contact area and the number of contact patches. For three typical rough surfaces with various mechanical properties, the present model gives accurate predictions of the load-area relation, which are verified by direct finite element simulations. An approximately linear load-area relation is observed for elastic-plastic contact up to a large contact fraction of $15 \%$, and the influence of yield stress is addressed.
\end{abstract}

Key words: Contact, Rough Surfaces, Elastic-Perfectly Plastic Solid, Contact Fraction 


\section{Introduction}

Prediction of the real contact area between realistic rough surfaces plays a pivotal part in many fields such as friction, wear, electric conduction and heat transfer, and thus has always been the focus of the academic community. Since the pioneering works by Archard [1] and Greenwood and Williamson (GW model) [2], much theoretical progress has been made over the past decades, including the elastic GW-based statistical models [3, 4] and the prominent scaling theory by Persson [5]. Comparisons between the multi-asperity models and Persson's theory were discussed [6]. The deformation mechanism at the nanoscale has been incorporated into the contact of random rough surfaces [7]. For rough surfaces with regular patterns, finite element method and theoretical model have also been conducted to analyze the influence of roughness on stiffness $[8,9]$. However, due to the complicated nature of surface topography and the distinct deformation mechanisms at various scales combined with the bulk deformation, the contact of rough surfaces is still an opening and challenging task [10].

Because of the multi-scale nature of rough surfaces, the real area in contact is usually a small fraction of the nominal surface area. The local contact pressure at many contact patches will go beyond the elastic limit, even under a very light load. Therefore, plastic deformation is indeed inevitable for the contact of rough surfaces. Bowden and Tabor [11] assumed a constant plastic flow pressure over contact area, whereby the linear load-area relation could be easily obtained. Based on the observation of volume conservation induced by plastic deformation and a constant 
contact pressure, Pullen and Williamson [12] adopted an energy balance method to determine the load-area relation for plastic contact of rough surfaces. The original GW model has been widely extended by applying proper elastic-plastic contact model to each isolated contacting asperity. For example, Chang et al. [13] furthered this model by considering the volume conservation of plastically deformed asperities. In view of the fractal characteristic of rough surfaces, Majumdar and Bhushan [14] presented a fractal contact model, in which contact patch with area larger than a critical value obeys the elastic Hertz theory, while that with smaller area is assumed deforming fully plastically. Inspired by Archard's model [1], Jackson and Streator [15] proposed an iterative multi-scale approach to calculate the contact area of elastic-plastic solids. Adopting the concept of scale magnification, Persson [16] accounted for the plasticity through introducing the yield stress as the upper bound of local contact pressure. Using an explicit dynamic Lagrangian finite element calculation, Pei et al. [17] modelled the contact between a rigid plane and an elasto-plastic solid with fractal surface. Scale dependent strain gradient plasticity has also been incorporated into the contact of rough surfaces [18]. Even with the presence of appreciable plastic deformation, these works displayed a linear rise of contact area with load. And plastic deformation always takes place throughout the contact process, which was observed in a recent experiment [19].

In addition to multi-asperity models, the profilometric model was introduced by Abbott and Firestone in 1933 [20]. In this model, the contact area was taken as the geometrical intersection of the rough surface by a virtual plane. For fully plastic 
deformation, the load was given by the contact area times the flow pressure or the indentation hardness [21]. When the contact area is an appreciable fraction of the apparent area, the whole concept of multi-asperity model fails, while the profilometric theory can still be employed to determine the contact area for realistic separation [22]. Recently, Wang et al. [23] adopted the profilometric model to calculate the contact area, and then used an incremental equivalent model to analyze the elastic contact of rough surfaces, which showed good agreement with direct finite element simulation. In the present work, this approach will be used for the contact of elastic-perfectly plastic solids with rough surfaces.

The paper is organized in the following order. In section 2 , the incremental equivalent contact model for rough surfaces is summarized. The indentation of elastic-perfectly plastic solids by a circular punch is simulated in section 3 to obtain the general expression of the contact stiffness. In section 4, the elastic-plastic contacts for three typical rough surfaces are modelled by finite element method. Finally, the comparisons between the predictions of new model and the finite element simulations are discussed in section 5 .

\section{The incremental equivalent contact model}

Recently, Wang et al. [23] developed an incremental elastic contact model for rough surfaces, in which the contact of rough surfaces was modelled by a group of equivalent circular contacts with varying radius. Here the basic concept and 
formulations of this model are presented briefly, and are adopted to deal with the elastic-plastic contact of rough surfaces.

Consider an elastic-perfectly plastic substrate with rough surface being compressed by a rigid plane, as schematically shown in Fig. 1(a). The Young's modulus of the substrate is $E$, the Poisson's ratio $v$ and the yield stress $\sigma_{\mathrm{y}}$. Choose the mean plane of the rough surface as the reference plane, and use the separation between the rigid plane and the reference plane indicating the compressive process.

Given the height information of a rough surface, the total section area $A_{\mathrm{c}}$ and the number of section patches $N$ generated by a virtual plane at a surface separation $z$ can be determined through geometrical analysis. The size and shape of these sections may be irregular as shown in Fig. 1(b). They are further simplified by $N$ identical circular contacts having the same total contact area $A_{\mathrm{c}}$, as shown in Fig. 1(c). Thus, the equivalent radius of contacts is given by

$$
R(z)=\left[\frac{A_{\mathrm{c}}(z)}{\pi N(z)}\right]^{1 / 2}
$$

In this model, the purely geometrical generated section area will be treated as real contact area, and then it is to determine the load required to achieve such contact area reversely by an incremental form.

At a specific surface separation $z$, denote the current load by $P$ as shown in Fig. 1 . Then for a decrement of separation $\mathrm{d} z$, we try to calculate the required increment of load $\mathrm{d} P$ using the contact stiffness defined by $\mathrm{d} P / \mathrm{d} z$. For a circular contact of radius $R$, the contact stiffness in elastic deformation is given by $2 E^{*} R$ with $E^{*}=E /\left(1-v^{2}\right)$ being the plain-strain elastic modulus of the substrate [24]. While for elastic-perfectly 
plastic deformation, the contact stiffness is a little complicated, but can be expressed in terms of the current load, the yield stress $\sigma_{\mathrm{y}}$ in addition to $E^{*}$ and $R$ to be given in section 3 .

For $N$ circular contacts with identical radius $R$, the total contact stiffness is given as

$$
\frac{\mathrm{d} P}{\mathrm{~d} z}=g\left(\frac{P / N}{\pi R^{2} \sigma_{\mathrm{y}}}\right) 2 N E^{*} R
$$

in which $g$ is a dimensionless function given in section 3. Substitution of Eq. (1) into Eq. (2) gives

$$
\frac{\mathrm{d} P}{\mathrm{~d} z}=g\left(\frac{P}{A_{\mathrm{c}} \sigma_{\mathrm{y}}}\right) \frac{2 E^{*}}{\sqrt{\pi}}\left(A_{\mathrm{c}} N\right)^{1 / 2} .
$$

The initial load is taken as $P=0$ for the surface separation $z$ at infinity. To achieve a contact area $A_{\mathrm{c}}(z)$, the total load is obtained by solving the differential equation (3).

The present model demands both the contact area $A_{\mathrm{c}}(z)$ and the number of contact patches $N(z)$. From the Abbot-Firestone curve [20], $A_{\mathfrak{c}}(z)$ can be immediately extracted. However, the number of contact patches $N(z)$ is too complicated to be given in an explicit expression [21]. As an option, numerical techniques are adopted to obtain both $A_{\mathfrak{c}}(z)$ and $N(z)$ from rough topographies in the present study.

In our operations, a rough surface is discretized by nodes with identical lateral spacing, then each node contributes equally to the projected nominal contact area $A_{0}$. For a given surface separation $z$, any node with a height higher than $z$ are marked as one contact node. Then the contact fraction $A_{\mathrm{c}}(z) / A_{0}$ can be calculated as the ratio of the number of contact nodes to the amount of surface nodes. Meanwhile, any two 
surface nodes with their projections on the reference plane being adjacent are recognized as within the same contact patch, then the amount of the contact patches $N(z)$ can be obtained.

Eq. (3) is a differential equation, and the contact stiffness $\mathrm{d} P / \mathrm{d} z$ is a function of $z$ and $P$ itself. At a sufficiently large surface separation $z$, the normal load $P$ vanishes. Such an initial value problem could be solved numerically by explicit iteration methods as the fourth-order Runge-Kutta method. Firstly, the surface separation $z$ is discretized as $z_{n}$ with identical interval $h$ in a descending order, and $z_{0}$ is large enough such that no contact happens and $P\left(z_{0}\right)=0$. Then, by using Eq. (3), the contact stiffness $\mathrm{d} P / \mathrm{d} z$ at points $\left(z_{n}, P_{n}\right),\left(z_{n}+h / 2, P_{n}+k_{1} h / 2\right),\left(z_{n}+h / 2, P_{n}+k_{2} h / 2\right)$ and $\left(z_{n}+h\right.$, $\left.P_{n}+k_{3} h\right)$ are calculated successionally as $k_{1}, k_{2}, k_{3}$ and $k_{4}$, respectively. As surface separation decreasing from $z_{n}$ to $z_{n+1}$, the load increment $P_{n+1}-P_{n}$ is then calculated as $\left(k_{1}+2 k_{2}+2 k_{3}+k_{4}\right) / 6$ times the interval $h$. Finally, the load sequence $P_{n}$ and the subsequent load-area relation are obtained. The interval of surface separation $h$ is chosen as 0.002 times the root-mean-square (rms) roughness $\sigma$ of the rough surface. Since the numerical method adopted here is a fourth-order method, the total accumulated error of load $P$ is on the order of $o\left[(0.002 \sigma)^{4}\right]$, which is negligible.

\section{Contact stiffness of elastic-perfectly plastic substrate}

Riccardi and Montanari [25] summarized the existing works on indentations of elastic-plastic metals by a rigid flat punch, and gave an approximate load-depth relation for deep indentation. In our model, a general expression of the contact 
stiffness is preferred to covering both the elastic and plastic regimes. Therefore, in this section, finite element simulations are performed to determine the contact stiffness. The flat-ended cylindrical indentation on an isotropic elastic-perfectly plastic substrate is simulated, which keeps a constant contact radius. Parametric analysis is adopted to summarize the general contact stiffness with respect to material properties and geometric size.

The finite element simulation is conducted through the commercial software ABAQUS. The finite element model is shown in Fig. 2. The indenter is modeled by a rigid cylinder with radius $R$. At the edge of the indenter, fillet is adopted to reduce stress concentration. The radius of the fillet is set to be $1 \%$ of the contact radius, which has negligible effect to the overall indentation response.

The substrate is set to be elastic-perfectly plastic. The Young's modulus $E$ is chosen as $100 \mathrm{GPa}$, and Poisson's ratio $v$ equals 0.3 . The yield stress $\sigma_{\mathrm{y}}$ takes values of $100 \mathrm{MPa}, 400 \mathrm{MPa}, 700 \mathrm{MPa}$ and $1 \mathrm{GPa}$, respectively. The ratio $\sigma_{\mathrm{y}} / E$ varies from 0.001 to 0.01 , which are typical for metals. Both the radius and height of the substrate are $120 R$, which are large enough to rule out the boundary effect.

Three-node linear axisymmetric triangle elements are used to discretize the substrate, with refined meshes near the contact region. The symmetric boundary conditions are applied at $r=0$, while free boundary condition is assumed at the outer radius of the substrate. The displacement along $z$-axis is restricted at the bottom of the substrate. Frictionless contact is assumed between the indenter and the substrate. Adaptive mesh method is applied to maintain mesh quality during the indentation 
process. The accuracy of numerical results has been guaranteed through convergence tests.

According to Riccardi and Montanari's analysis, the overall contact response can be described by the variation of the dimensionless load $L /\left(\pi \sigma_{\mathrm{y}} R^{2}\right)$ with respect to the normalized indentation depth $2 E^{*} \delta /\left(\pi \sigma_{\mathrm{y}} R\right)$. For the indentations on various substrates, it is found that the load-depth relation does follow this scaling law, as shown in Fig. 3. When the mean pressure $p=L /\left(\pi R^{2}\right)$ is below the yield stress, the linear relation holds between the load and indentation depth. As the depth increases further, the curve will deviate from the linear elastic relation and the slope will drop. At very large depth, the mean pressure maintains almost a constant (i.e. $3 \sigma_{\mathrm{y}}$ ). The general load-depth relation can be fitted by

$$
\frac{L}{\pi R^{2} \sigma_{\mathrm{y}}}=f\left(\frac{2 E^{*} \delta}{\pi \sigma_{\mathrm{y}} R}\right),
$$

in which the function $f(x)$ is given by

$$
f(x)=x\left(1+0.0832 x^{3.23}\right)^{-0.239}
$$

Based on this relation Eq. (4), the contact stiffness of an elastic-perfectly plastic substrate with a circular contact radius $R$ can be obtained as

$$
\frac{\mathrm{d} L}{\mathrm{~d} \delta}=g\left(\frac{L}{\pi R^{2} \sigma_{\mathrm{y}}}\right) 2 E^{*} R
$$

For the convenience for later use, the function $g(x)$ can be simplified as

$$
g(x)=\left(1+0.0415 x^{4.50}\right)^{-1.40}
$$

\section{Finite element simulation of rough surface contact}


For the sake of testing the efficiency of our model, the contact process of a rigid plane and an elastic-perfectly plastic substrate with various rough surfaces is simulated through ABAQUS, as shown in Fig. 4. The projection of the rough surface of the substrate is square with a length $l$, and the height of the substrate is $l / 4$. The elastic properties of the substrate are chosen as $E=100 \mathrm{GPa}$ and $v=0.3$. Various yield stresses $\sigma_{\mathrm{y}}$ are taken as $100 \mathrm{MPa}, 400 \mathrm{MPa}, 700 \mathrm{MPa}$ and $1 \mathrm{GPa}$ to analyze the influence of plastic deformation.

$256 \times 256$ nodes with identical lateral spacing are generated on the upper surface of the substrate to produce rough surfaces. Then, eight-node linear hexahedral (C3D8I) elements are generated with respect to these surface nodes, supported by layers of gradually coarsen ten-node quadratic tetrahedral (C3D10) elements. Again, C3D8I elements are used to construct the rest of the substrate, as shown in Fig. 4. Frictionless contact is assumed between the substrate and the rigid plane.

Simulations are performed for three rough surfaces of different kinds, as shown in Fig. 5. Surface A and surface B are artificially generated using an open source code in MATLAB [26], whereas surface $\mathrm{C}$ is measured from a ground metallic sample with a white light interferometer. The former two surfaces are isotropic, with their axisymmetric surface power spectrum density functions $C(q)$ shown in Fig. 6. The power spectrum of surface $A$ is self-affine fractal on all length scale with Hurst exponent $H=0.5$, but only in high frequency span dose surface B maintains the fractal characteristic with $H=0.8$. As for surface $\mathrm{C}$, its anisotropic feature is prominent, and $C(q)$ varies in directions parallel or perpendicular to the gullies. The 
lengths $l$ of surface A and surface B are $200 \mu \mathrm{m}$, and $l$ takes $52.2 \mu \mathrm{m}$ for surface C. The rms roughness $\sigma$ is calculated as $0.500 \mu \mathrm{m}, 1.00 \mu \mathrm{m}$ and $0.103 \mu \mathrm{m}$ for surface A, $\mathrm{B}$ and $\mathrm{C}$, respectively.

\section{Results and discussions}

For those three rough surfaces, we use the numerical approach in section 2 to calculate the contact fraction $A_{\mathrm{c}} / A_{0}$ and the amount of contact patches $N$, as displayed in Fig. 7. As the surface separation $z / \sigma$ decreases, the contact area continuously increases, whereas the density of the contact patches varies in a fluctuant way, implying the coalescing of contact patches at small separation.

Then both the model in section 2 and the finite element method in section 4 are employed to calculate the load-area relation, as shown in Figs. 8-10 for surfaces A, B and $\mathrm{C}$, respectively. In these figures, the lines represent the predictions by our proposed model, whereas the scatters indicate the finite element simulations. This notation will also be used in the following figures. Up to a small separation with the contact fraction being $15 \%$, an approximately linear relation between external load and contact area is predicted for both purely elastic contact and elastic-perfectly plastic contact. More importantly, our model agrees well with the finite element simulations for three different rough surfaces. Compared with the purely elastic contact at a given load, the contact area will increase when plastic deformation is considered. The smaller the yield stress, the larger the contact area.

The nearly linear behavior of load-area curves implies an approximate constant 
mean pressure over the rough surfaces. Fig. 11 displays the variation of the mean pressure $P / A_{\mathrm{c}}$ normalized by the plain-strain elastic modulus $E^{*}$ with respect to the contact fraction. For small contact fraction less than $1 \%$, statistical fluctuations appear, in which the total number of contact nodes is quite small and the mean normal pressure is quite sensitive to the contact area. When the contact fraction is larger than $1 \%$, the mean pressure gets stable throughout the contact process. Since the beginning of contact, the mean pressure is beyond the yield stress. For examples, the mean pressure is about $2.5 \sigma_{\mathrm{y}}$ to $3.5 \sigma_{\mathrm{y}}$ for material with $\sigma_{\mathrm{y}} / E=0.001$, and is about $2 \sigma_{\mathrm{y}}$ to $2.5 \sigma_{\mathrm{y}}$ for substrate with $\sigma_{\mathrm{y}} / E=0.007$. Therefore, plastic deformation takes place throughout the contact process of rough surfaces, which is clearly distinct from the indentation by a single punch with elastic deformation firstly and plastic deformation subsequently in section 3. Recently, experiments on metals have verified this characteristic [19]. Fig. 11 demonstrates that the magnitude of mean pressure depends on the mechanical properties and topography of rough surface. With the yield stress enhanced, the mean pressure will rise.

For a given contact fraction (i.e. 5\%), the dependence of mean pressure on yield stress is plotted in Fig. 12. For a specific rough surface, the mean pressure is bounded by an upper limit corresponding to purely elastic contact. While for fully plastic contact at small $\sigma_{\mathrm{y}} / E^{*}$, the mean pressure is often given by the hardness of substrate, which is about 3 times the yield stress $[11,21]$. For a high yield stress, the mean pressure moves to the elastic limit and the topography plays an evident effect. While for a low yield stress, the influence of topography becomes weak. For $\sigma_{\mathrm{y}} / E^{*}$ under 
0.01 , the relation of mean pressure to yield stress can be described by a power law $P / A_{\mathrm{c}} \propto \sigma_{\mathrm{y}}^{0.85}$. It seems that for three distinct rough surfaces, the power index is almost the same. For fractal rough surfaces, the finite element simulations of Pei et al. exhibited a similar feature [17].

\section{Conclusions}

In this paper, we develop a new method to determine the load-area relation for contact of elastic-perfectly plastic solids with rough surfaces. For various material properties and rough surfaces, the predictions of our model agree reasonable well with direct finite element simulations. Even for elastic-plastic contact, the contact area is linearly proportional to the load till for a large contact fraction as $15 \%$. Since the beginning of contact, the plastic deformation plays its role and tends to increase contact area. The mean pressure is beyond the yield stress at the initial contact and keeps almost constant throughout the contact process. The enhancement of yield stress could improve the mean pressure, following a power law relation. The new proposed model demonstrates its accuracy, conciseness and general applicability, and is a powerful tool to deal with contact of rough surfaces.

\section{Acknowledgements}

Supports from the National Natural Science Foundation of China (Grant No. 11525209) are acknowledged. 


\section{References}

[1] Archard, J. F. (1957). Elastic Deformation and the Law of Friction. Proceedings of the Royal Society A-Mathematical, Physical and Engineering Sciences, 243(1233), 190-205.

[2] Greenwood, J. A., \& Williamson, J. B. P. (1966). Contact of Nominally Flat Surfaces. Proceedings of the Royal Society A-Mathematical, Physical and Engineering Sciences, 295(1442), 300-319.

[3] Bush, A. W., Gibson, R. D., \& Thomas, T. R. (1975). The Elastic Contact of a Rough Surface. Wear, 35(1), 87-111.

[4] Greenwood, J. A. (2006). A Simplified Elliptic Model of Rough Surface Contact. Wear, 261(2), 191-200.

[5] Persson, B. N. J. (2001). Theory of Rubber Friction and Contact Mechanics. Journal of Chemical Physics, 115(8), 3840-3861.

[6] Carbone, G., \& Bottiglione, F. (2008). Asperity Contact Theories: Do They Predict Linearity Between Contact Area and Load? Journal of the Mechanics and Physics of Solids, 56(8), 2555-2572.

[7] Yuan, W. K., Long, J. M., Ding, Y., \& Wang, G. F. (2018). Statistical Contact Model of Rough Surfaces: The Role of Surface Tension. International Journal of Solids and Structures, 138(1), 217-223.

[8] Jiang, W. G., Su, J. J., \& Feng, X. Q. (2008). Effect of Surface Roughness on Nanoindentation Test of Thin Films. Engineering Fracture Mechanics, 75(17), 4965-4972. 
[9] Li, S., Yao, Q. Z., Li, Q. Y., Feng, X. Q., \& Gao, H. J. (2018). Contact Stiffness of Regularly Patterned Multi-Asperity Interfaces. Journal of the Mechanics and Physics of Solids, 111, 277-289.

[10]Müser, M. H., Dapp, W. B., Bugnicourt, R., Sainsot, P., Lesaffre, N., Lubrecht, T. A., et al. (2017). Meeting the Contact-Mechanics Challenge. Tribology Letters, 65(4), 118.

[11]Bowden, F. P., \& Tabor, D. (1939). The Area of Contact Between Stationary and Between Moving Surfaces. Proceedings of the Royal Society A-Mathematical, Physical and Engineering Sciences, 169(938), 391-413.

[12]Pullen, J., \& Williamson, J. B. P. (1972). Plastic Contact of Rough Surfaces. Proceedings of the Royal Society A-Mathematical, Physical and Engineering Sciences, 327(1569), 159-173.

[13]Chang, W. R., Etsion, I., \& Bogy, D. B. (1987). An Elastic-Plastic Model for the Contact of Rough Surfaces. Journal of Tribology-Transactions of the ASME, 109(2), 257-263.

[14]Majumdar, A., \& Bhushan, B. (1991). Fractal Model of Elastic-Plastic Contact Between Rough Surfaces. Journal of Tribology-Transactions of the ASME, 113(1), 1-11.

[15]Jackson, R. L., \& Streator, J. L. (2006). A Multi-Scale Model for Contact Between Rough Surfaces. Wear, 261(11), 1337-1347.

[16]Persson, B. N. J. (2001). Elastoplastic Contact Between Randomly Rough Surfaces. Physical Review Letters, 87(11), 116101. 
[17]Pei, L., Hyun, S., Molinari, J. F., \& Robbins, M. O. (2005). Finite Element Modeling of Elasto-Plastic Contact Between Rough Surfaces. Journal of the Mechanics and Physics of Solids, 53(11), 2385-2409.

[18]Song, H., Van der Giessen, E., \& Liu, X. (2016). Strain Gradient Plasticity Analysis of Elasto-Plastic Contact Between Rough Surfaces. Journal of the Mechanics and Physics of Solids, 96, 18-28.

[19]Li, L. T., Liang, X. M., Xing, Y. Z., Yan, D., \& Wang, G. F. (2021). Measurement of Real Contact Area for Rough Metal Surfaces and the Distinction of Contribution From Elasticity and Plasticity. Journal of Tribology-Transactions of the ASME, 143(7), 071501.

[20]Abbott, E. J., \& Firestone, F. A. (1933). Specifying Surface Quality - A Method Based on Accurate Measurement and Comparison. Journal of Mechanical Engineering, 55, 569-572.

[21]Nayak, P. R. (1973). Random Process Model of Rough Surfaces in Plastic Contact. Wear, 26(3), 305-333.

[22] Greenwood, J. A. (1967). The Area of Contact Between Rough Surfaces and Flats. Journal of Lubrication Technology, 89(1), 81-87.

[23]Wang, G. F., Liang, X. M., \& Yan, D. (Preprint). (DOI: 10.13140/RG.2.2. 28161.89443)

[24]Sneddon, I. N. (1946). Boussinesq Problem for a Flat-Ended Cylinder. Proceedings of the Cambridge Philosophical Society, 42(1), 29-39. 
[25]Riccrdi, B., \& Montanari, R. (2004). Indentation of Metals by a Flat-Ended Cylindrical Punch. Materials Science and Engineering A, 381, 281-291.

[26]Kanafi, M. M. (2020). Surface Generator: Artificial Randomly Rough Surfaces (https://www.mathworks.com/matlabcentral/fileexchange/60817-surface-generato r-artificial-randomly-rough-surfaces), MATLAB Central File Exchange. Retrieved September 30, 2020. 


\section{Figure captions}

Figure 1. A schematic diagram of the equivalent circular contact model

Figure 2. Finite element model of the contact between a flat-ended cylinder and an elastic plastic half space

Figure 3. The variation of the normalized load $L /\left(\pi \sigma_{\mathrm{y}} R^{2}\right)$ with respect to the dimensionless indentation depth $2 E^{*} \delta /\left(\pi \sigma_{\mathrm{y}} R\right)$

Figure 4. Finite element model of the contact between an elastic plastic substrate and a rigid plane

Figure 5. The topography of rough surfaces: (a) surface A, (b) surface B, (c) surface C Figure 6. The surface power spectrum density functions of surfaces A, B, and C Figure 7. The variations of contact area $A_{\mathrm{c}} / A_{0}$ and contact patches $N$ with respect to $z / \sigma$

Figure 8. The dimensionless load $P /\left(E^{*} A_{0}\right)$ as a function of the contact fraction $A_{\mathrm{c}} / A_{0}$ for surface A

Figure 9. The dimensionless load $P /\left(E^{*} A_{0}\right)$ as a function of the contact fraction $A_{\mathrm{c}} / A_{0}$ for surface B

Figure 10. The dimensionless load $P /\left(E^{*} A_{0}\right)$ as a function of the contact fraction $A_{\mathrm{c}} / A_{0}$ for surface $\mathrm{C}$

Figure 11. The variation of the dimensionless mean pressure $P /\left(E^{*} A_{\mathrm{c}}\right)$ with respect to the contact fraction $A_{\mathrm{c}} / A_{0}$

Figure 12 . The variation of the dimensionless mean pressure $P /\left(E^{*} A_{\mathrm{c}}\right)$ with respect to the dimensionless yield stress $\sigma_{\mathrm{y}} / E^{*}$ 

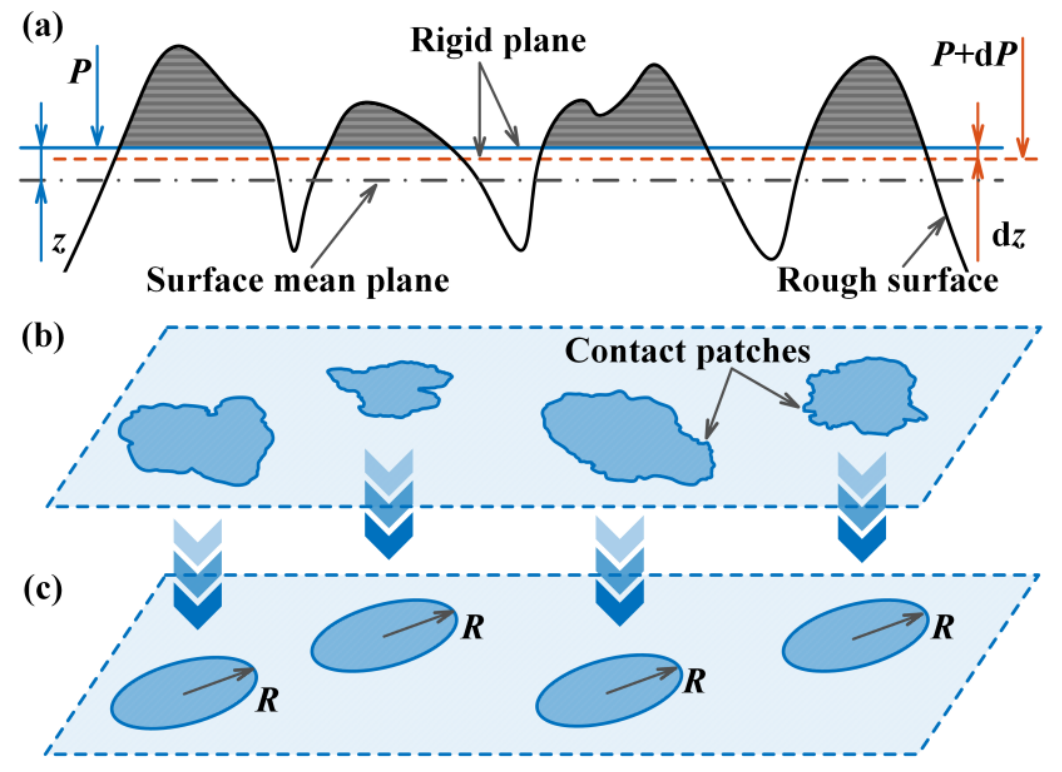

Fig. 1. 


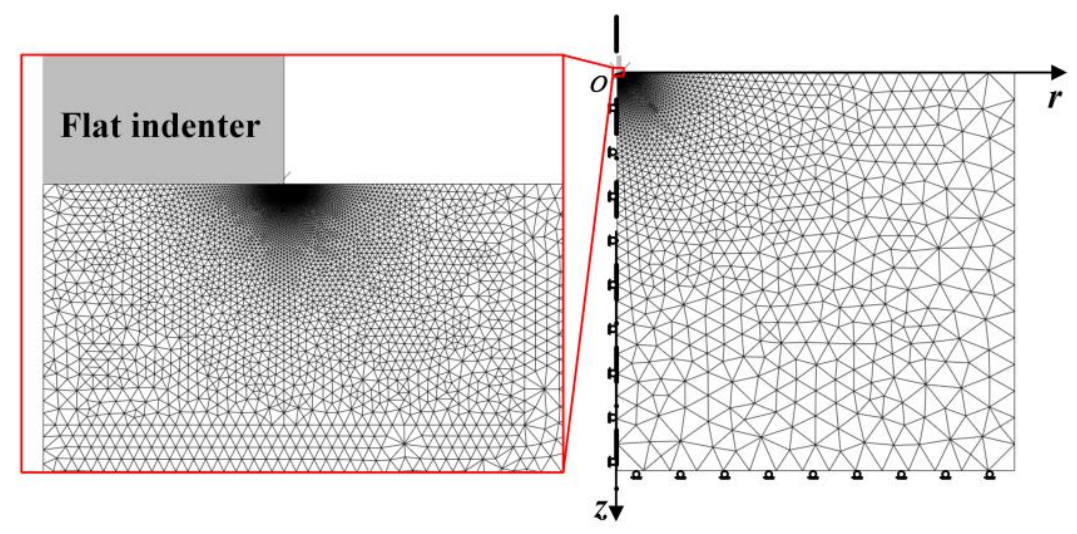

Fig. 2. 


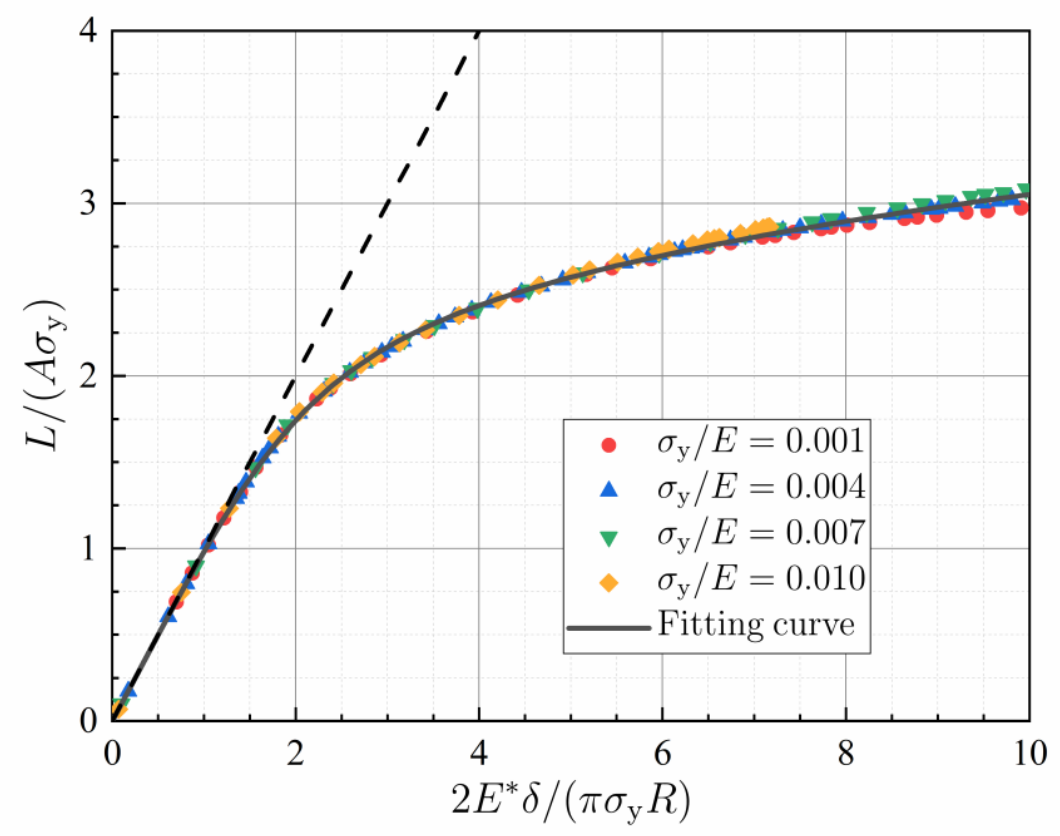

Fig. 3. 


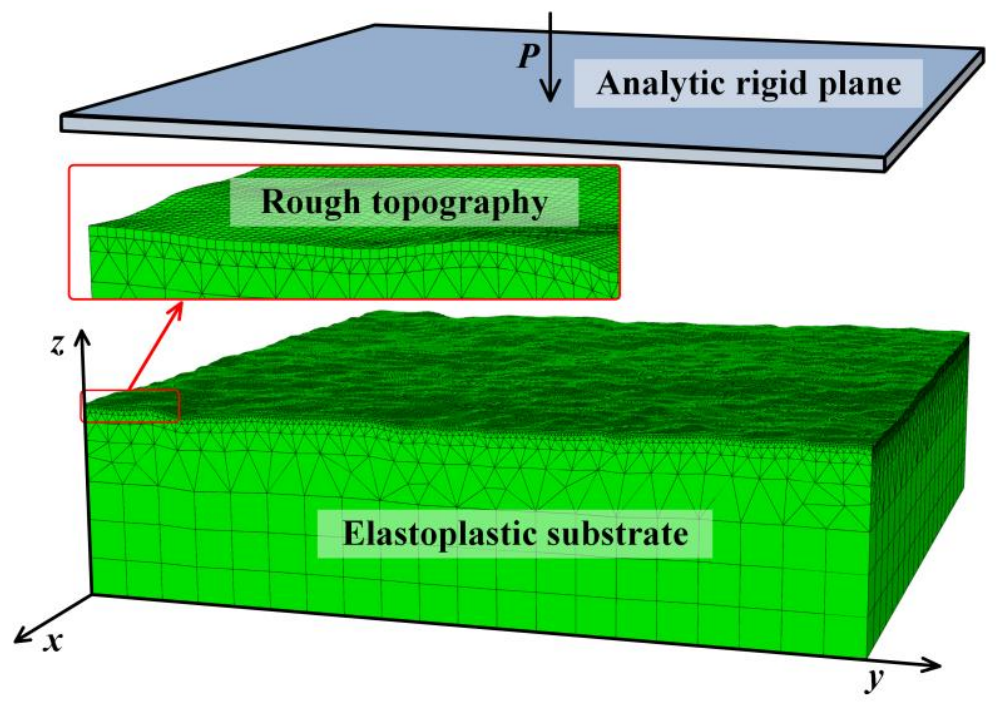

Fig. 4. 


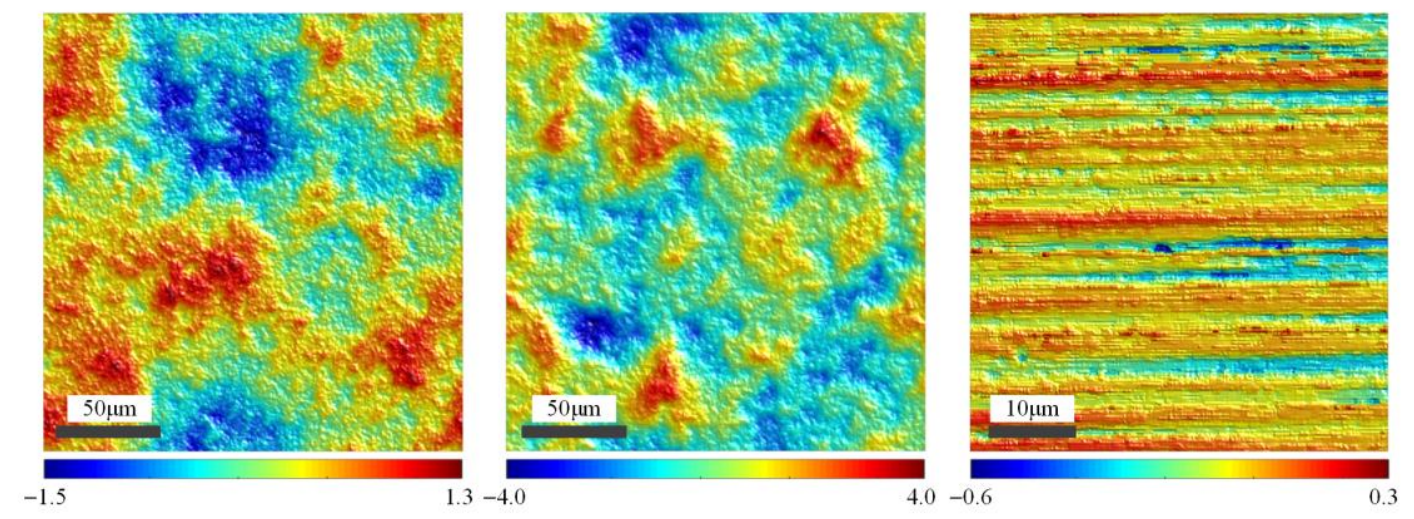

Fig. 5. 


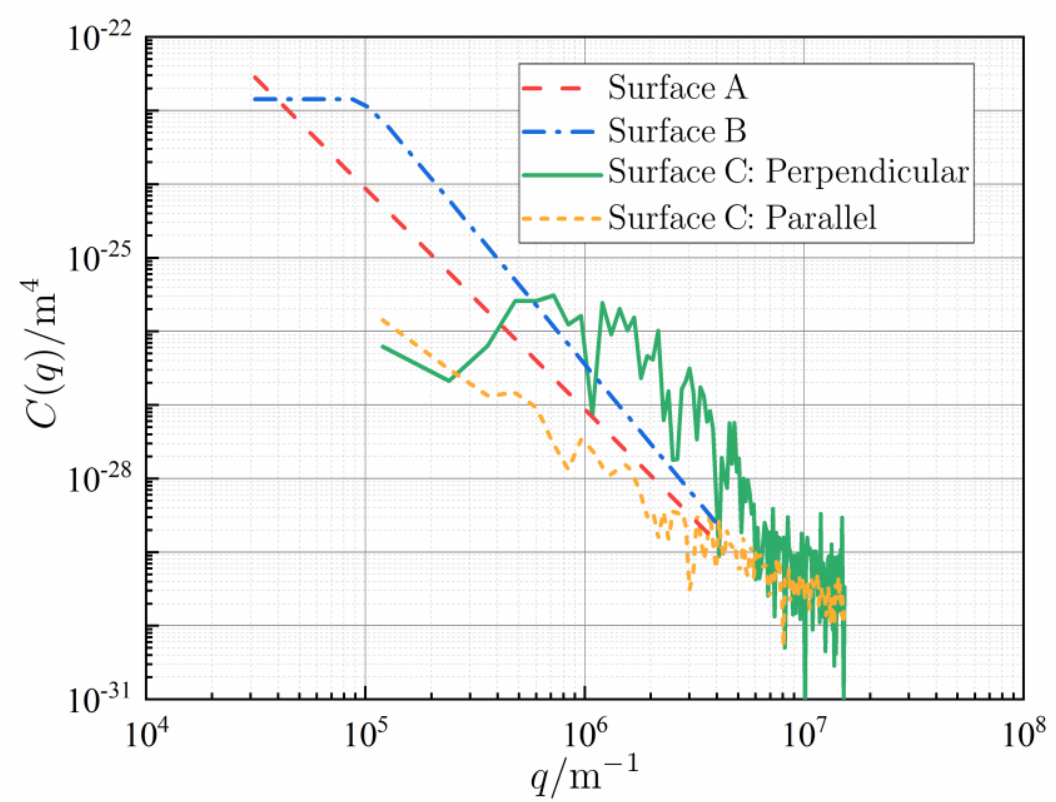

Fig. 6. 


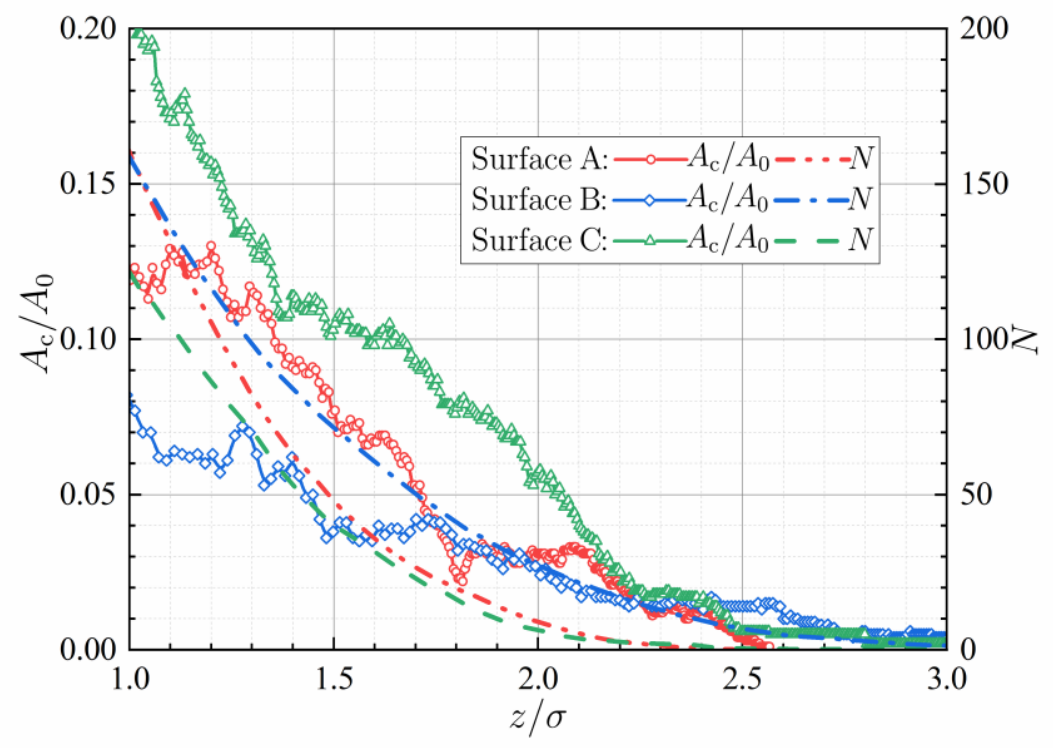

Fig. 7. 


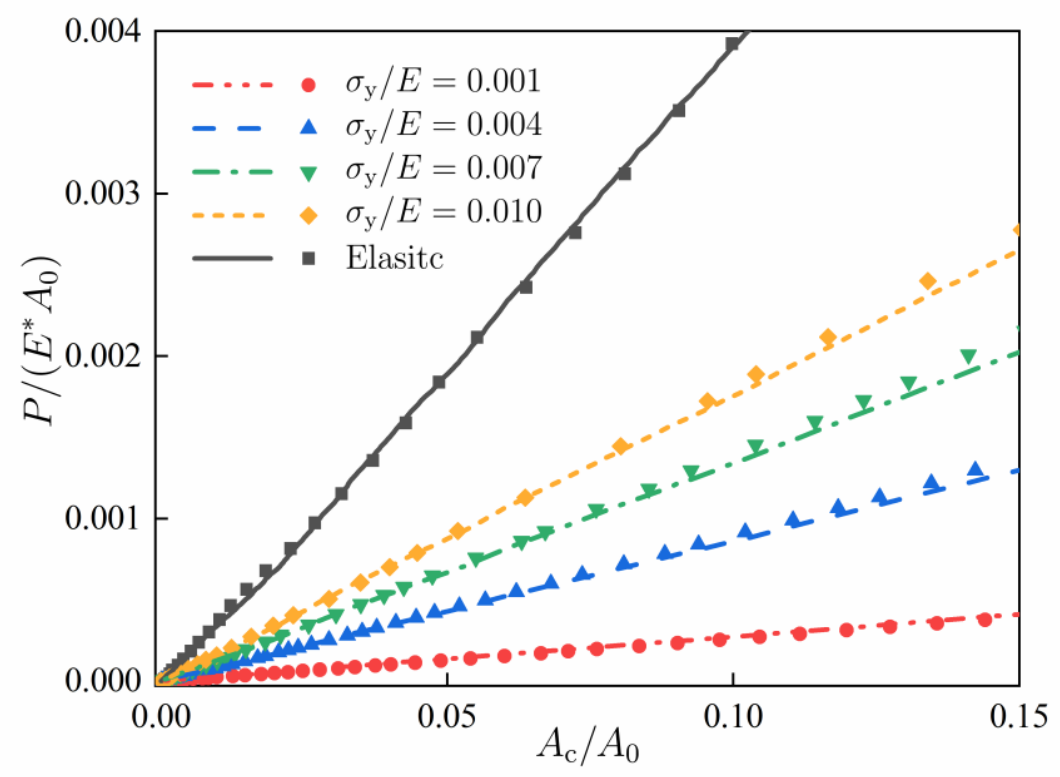

Fig. 8. 


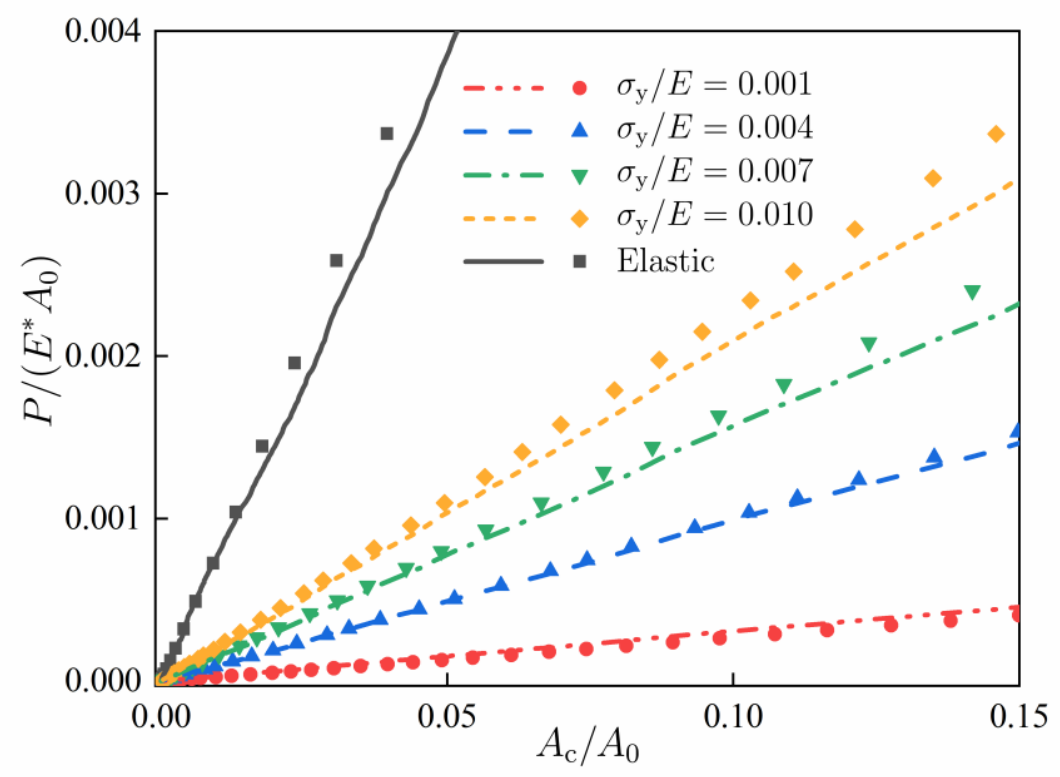

Fig. 9. 


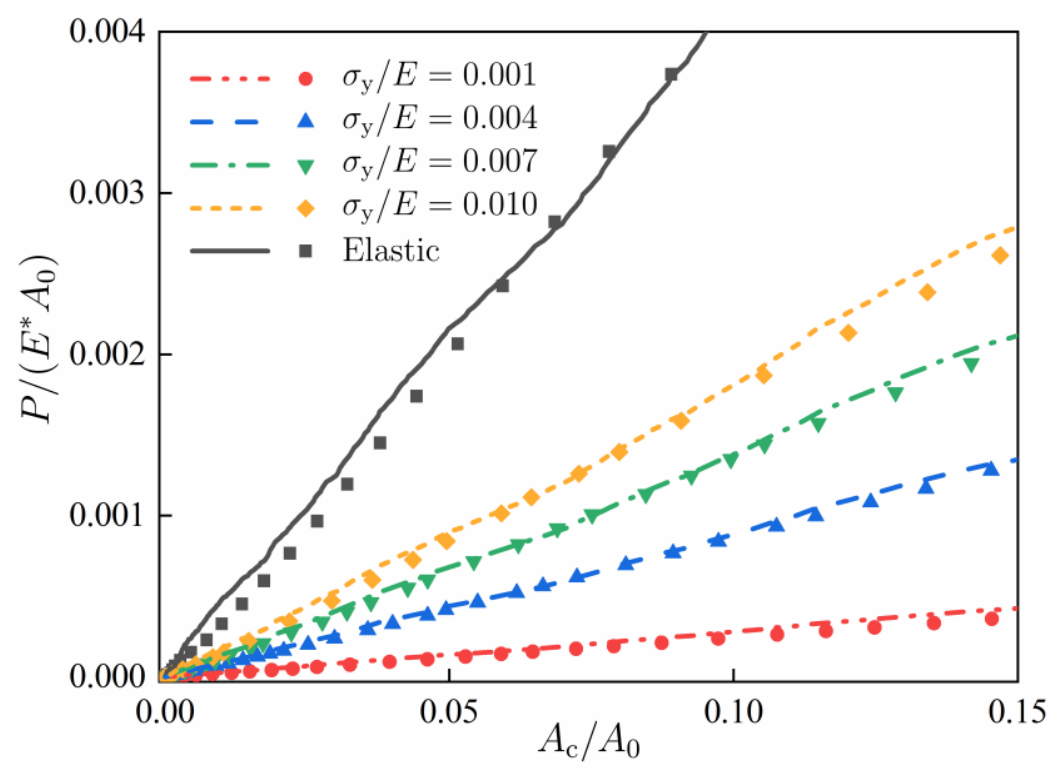

Fig. 10. 


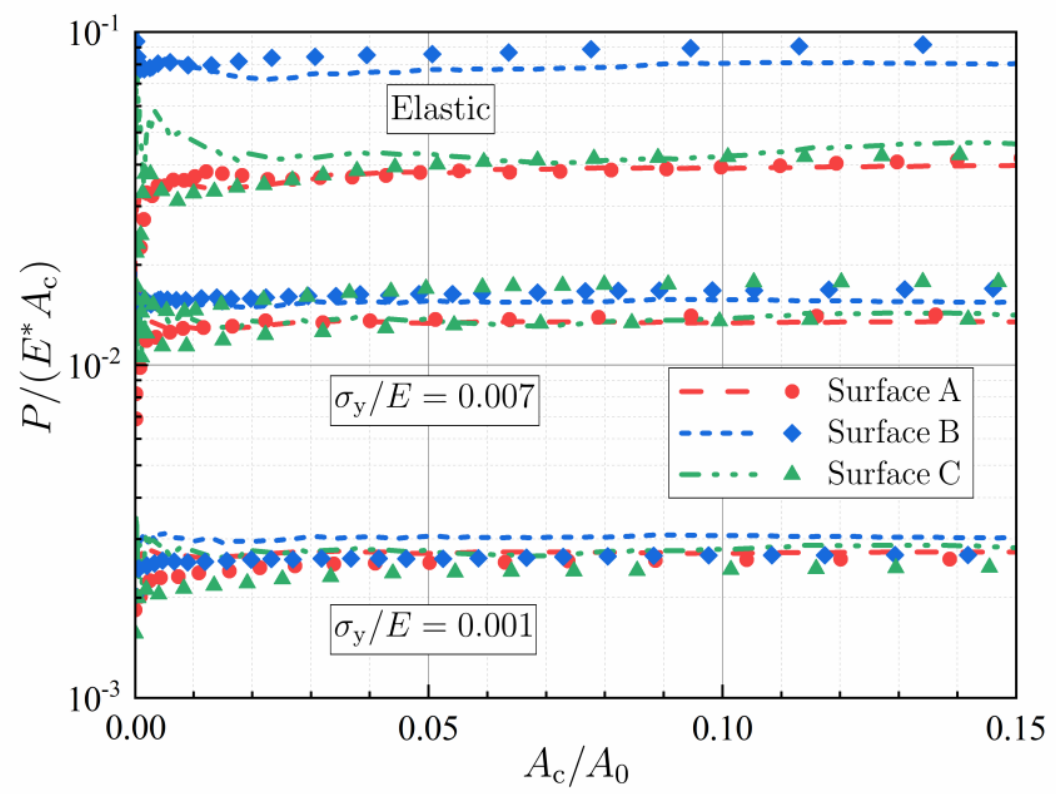

Fig. 11. 


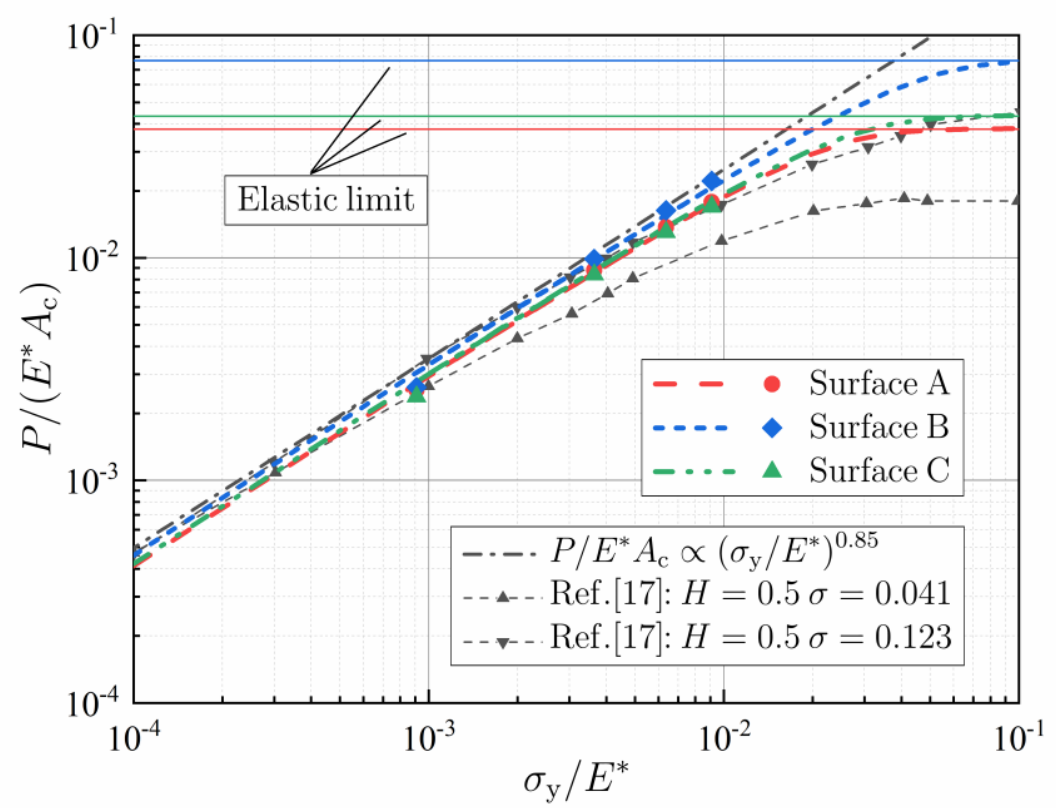

Fig. 12. 